MATEC Web of Conferences 47, 03021 (2016)

DOI: $10.1051 /$ matecconf/20164703021

(C) Owned by the authors, published by EDP Sciences, 2016

\title{
The Study of Soil-Roots Strength Performance by Using Pennisetum Setaceum Grass
}

\author{
Nor Azizi Yusoff ${ }^{1}$, Mohamad Fazrin Mohamad Fauzi ${ }^{1}$ and Firdaurs Abdullah ${ }^{1, a}$ \\ ${ }^{1}$ Faculty of Civil and Environmental Engineering, Universiti Tun Hussein Onn Malaysia, 86400 Parit Raja, Johor, \\ Malaysia
}

\begin{abstract}
Vegetation is regarded as a new engineering material contributes to slope stability and reduces superficial soil erosion, both by soil shear reinforcement and influencing soil water content. Yet, it is aware that roots growth occurred below the soil surface and it is considered as dynamic organs that respond to physical, chemical and hydrological induced by surrounding substrate. This study was carried out to investigate the soil shear strength performance by using Pennisetum Setaceum grass at the different planting period up to three months. The grass was planted in a $150 \mathrm{~mm} \times 200 \mathrm{~mm} \times 700 \mathrm{~mm}$ test plot with a suitable soil medium. Pull-out strength test was conducted for each plot to determine the soil pull-out strength according to different planting period. The results show that there is an increment in the pullout strength of rooted-soil sample over different planting period with average results of $6.7 \mathrm{kPa}, 11.4 \mathrm{kPa}$ and $25.8 \mathrm{kPa}$ for month 1,2 and 3 respectively. Based on this study, the soil pull-out strength developments for vegetated soil were observed. The higher pull-out strength was recorded for different growth period. Moreover, root weight was increased for different growth period. Based on the tensile test, the diameter of the root affects the rate of resistance against the tensile forces. This indicated that the roots structure growth affects the soil shear strength.
\end{abstract}

\section{Introduction}

Slope stabilization is the process of creating and implementing measures resistance to erosion and slope failure. The use of ground cover plants to overcome the slope failure was widely practiced throughout the world. Grass planting is the most effective method to stabilize the banks and slopes. The function of the grassroots is for gripping the particles of land slope and prevent it from slipping. It will be a strong vegetative barriers that slow the flow of water and filter and trap sediment in runoff [1]. In the event of rainfall on sloping surfaces, infiltration of rainwater into the slope will cause the pore water pressure increases, particularly near the surface of the slope. The increase in pore water pressure can make a decrease of shear strength, this facilitates the occurrence of failure. Through this study, infiltration of rainwater that weaken the strength of the slope near the surface will be examined in depth the effects of root growth on the slope in the context of slope stabilization. Interactions between plants and soil texture when planted with plants is carried out from time to time. In addition the strength of the performance of different construction periods has been investigated using the direct shear test machine and pull-out strength test. In addition, the strength test of the roots as incurred a

\footnotetext{
${ }^{\text {a }}$ Corresponding author : gf130019@siswa.uthm.edu.my
} 
tensile force was also studied in different diameters. Therefore, in this study, interaction between plants and soil as a result of roots growth in the context of the slope stabilization is studied. It was aimed to determine the pull-out strength performance of soil and Pennisetum Setaceum grass roots at different growth period of up to 3 months. Besides, the relationship between pull-out strength and tensile roots strength for vegetated soil at different growth period was examined. Knowing the influence of root tensile strengths towards the mechanical reinforcing effect, a tensile test has been conducted on the roots of Pennisetum setaceum grass.

\section{Materials and Methods}

\subsection{Plot preparation}

Four plots with dimension of $150 \mathrm{~mm}$ x $200 \mathrm{~mm}$ x $700 \mathrm{~mm}$ had been considered (Figure 1). All plots were planted with Pennisetum setaceum as shown in Figure 2(a). The grass was obtained from nearest nursery (Figure 2 (b)).

\subsection{Plot maintenance}

\subsubsection{Plant watering activities}

The grass uniformly was watered to ensure that the planted grass gets enough water to grow. However, the frequency of the plants watering normally affected by several factors such as the weather conditions, the maturity of the plant and plant species and soil types.

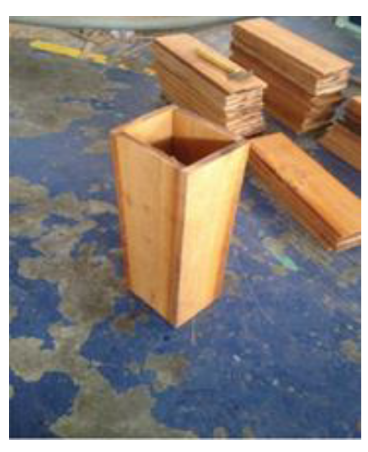

Figure 1. A wooden plot.
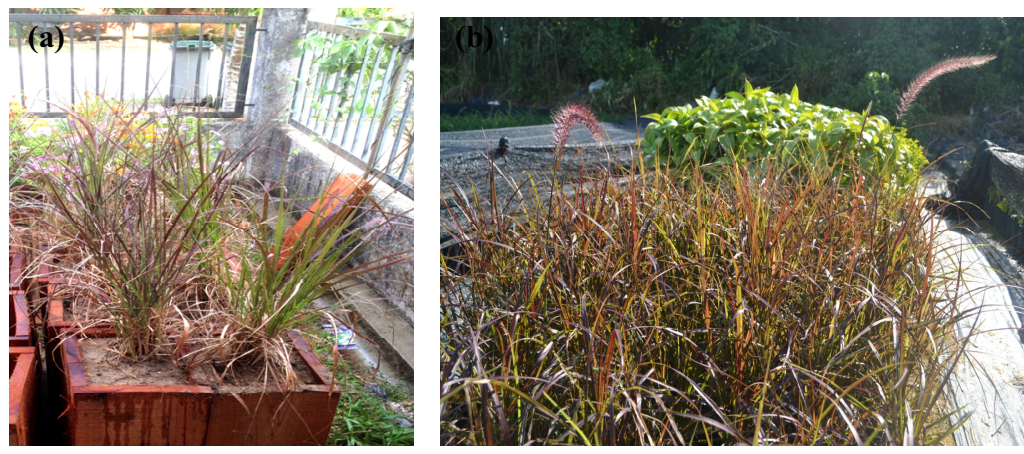

Figure 2. Guinea grass; (a) planted in plots and (b) nursery grown.

\subsubsection{Pull-out strength test procedure}

The selected plants that reached the required growth period were identified and marked using a tagging stick. Nine samples were selected for each of the same species (3 grasses for every month). Before setting up the pull-out equipment (Figure 3 (a)), the surface of the soil in the plot is cleared. Prior to the pull-out strength test, several factors that will affect the results must be carefully taken into consideration. For example, the equipment to be used must be in good condition, well-calibrated especially the portable scale. Pennisetum Steaceum grass clumps should be tied firmly using an adhesive tape and placed closer to portable scale that clung onto that grass clump (Figure 3 (c)). 


\subsection{Root screening process}

Once pull-out test is completed, the rooted sample is washed to remove soil from the clod leaving only the root specimens (Figure 4). This screening process enabled the root growth to be measured in term of mass at different planting period up to 3 months.



Figure 3. Pull-out strength test; (a) the pull-out machine, (b) test set-up, (c) portable scale being attached to grass clump and (d) Grass being completely pull-out from the soil.

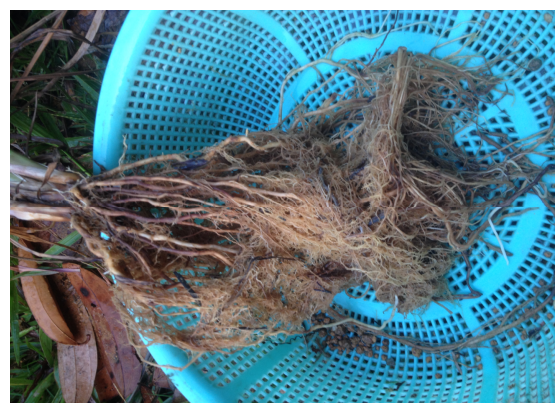

Figure 4. Root screening process, leaving only roots out of soil clod.

\subsection{Tensile test procedure}

Sample preparation is done by grouping the grass roots according to diameter size. Every root specimen is cut at the same length of $10 \mathrm{~mm}$ (Figure 5 (b)). This is done to ensure that the roots are in the uniform span to avoid error. To measure the root diameter, TMI Precision Micrometer (Figure 5(c)) had been used because it's capable to read up to 4 decimal places of diameter accuracy compared with common vernier calipers. Tensile strength of roots was determined using testing machine Testometric Micro 350 (Figure 5 (d)).

\section{Results and Discussion}

\subsection{Pull-out test}

Figure 6(a) shows the maximum pull-out strength for of Pennisetum Setaceum grass samples in the first month, which is $6.500 \mathrm{kPa}$, and $6.911 \mathrm{kPa}$. Average maximum pull-out strength of these two data is represented by a curve in Figure 6(b). There are only two data recorded for that month due to another sample indeliberately destroyed. For the second month planting period, the maximum pull-out strength recorded are $11.470 \mathrm{kPa}, 11.263 \mathrm{kPa}$ and $11.592 \mathrm{kPa}$ (Figure 6(c)). The average maximum 
pull-out strength of month 2 is shown in Figure 6(d). Lastly, on the third month, results recorded on maximum pull-out strength for three sample of grass are $20.590 \mathrm{kPa}, 28.680 \mathrm{kPa}$ and $28.080 \mathrm{kPa}$ (Figure 6(e)). Theirs average is shown in Figure 6(f).

Figure 7 shows the increment trend of the average pull-out strength from first month to third month of Pennisetum setaceum. The data shows that there has been an increase of pull-out strength from the first to the third month which is $6.705 \mathrm{kpa}, 11.442 \mathrm{kpa}$ and $25.783 \mathrm{kpa}$ for month 1,2 and 3 respectively.
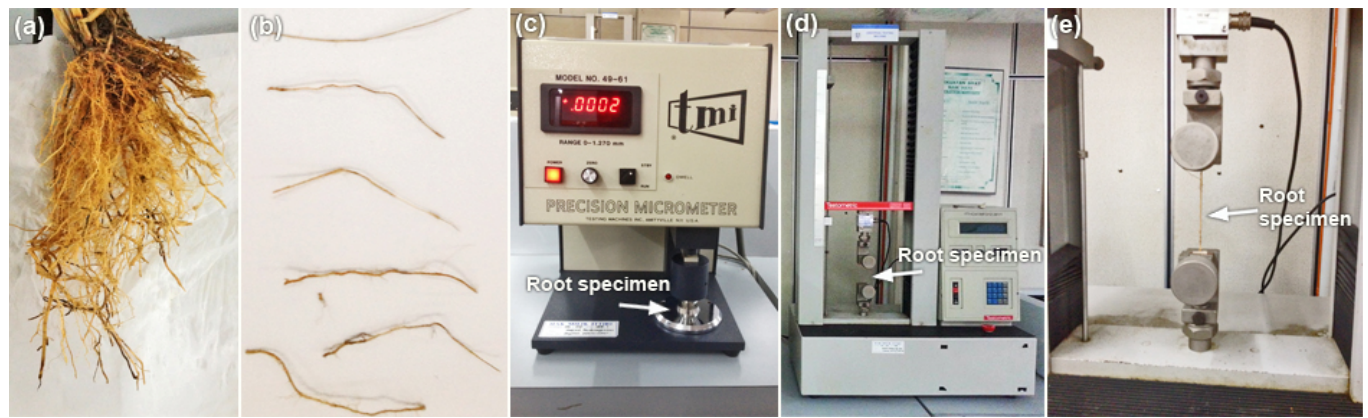

Figure 5. Root tensile test; (a) roots after being washed, (b) roots were cut into same length, (c) root diameter is measured using TMI Precision Micrometer, (d) Testometric Micro 350 and (e) root specimen is being clamped by a pair of wedge grip.

\subsection{Pull-out strength comparison over time}

The comparisons performed on the results of pull-out tests for different planting period showed a linear increment for the average pull-out strength encompassed different planting period (Figure 8).

\subsection{Effect of root weight on pull-out strength}

As expected at the beginning of this study, soil pull-out strength increased with the growth of roots [2]. However, based on Figure 9, this study showed mass of roots only increased drastically on month 2 compared to month 1 . It is noticed very small increase in mass of root for month 3 compared to month 2. The same pattern of increment also observed in the graph of root mass against average pullout strength (Figure 10).

\subsection{Tensile strength}

Result of the maximum tensile resistance of roots in diameter ranged from 0.28 to $1.36 \mathrm{~mm}$ shows that Pennisetum grass exhibited the maximum tensile force of $22 \mathrm{~N}$ (Figure 11). Hence, based on the observation of graph maximum tensile resistance versus diameter, an increase in diameter would increase the tensile resistance. Similar trend was also observed by [2, 3]. Finally, the maximum tensile strength (force/root area) of Pennisetum grass was $279 \mathrm{MPa}$ (Figure 12). From the regression analysis between tensile strength and root diameter, it can be observed that the tensile strength decreased with increasing root diameter which is in line with the previous root studies conducted by [2, 4]. According to [5], during soil shearing, the finer and thinner roots that have high tensile strength would slip while the thicker roots with lower tensile strength would break. 

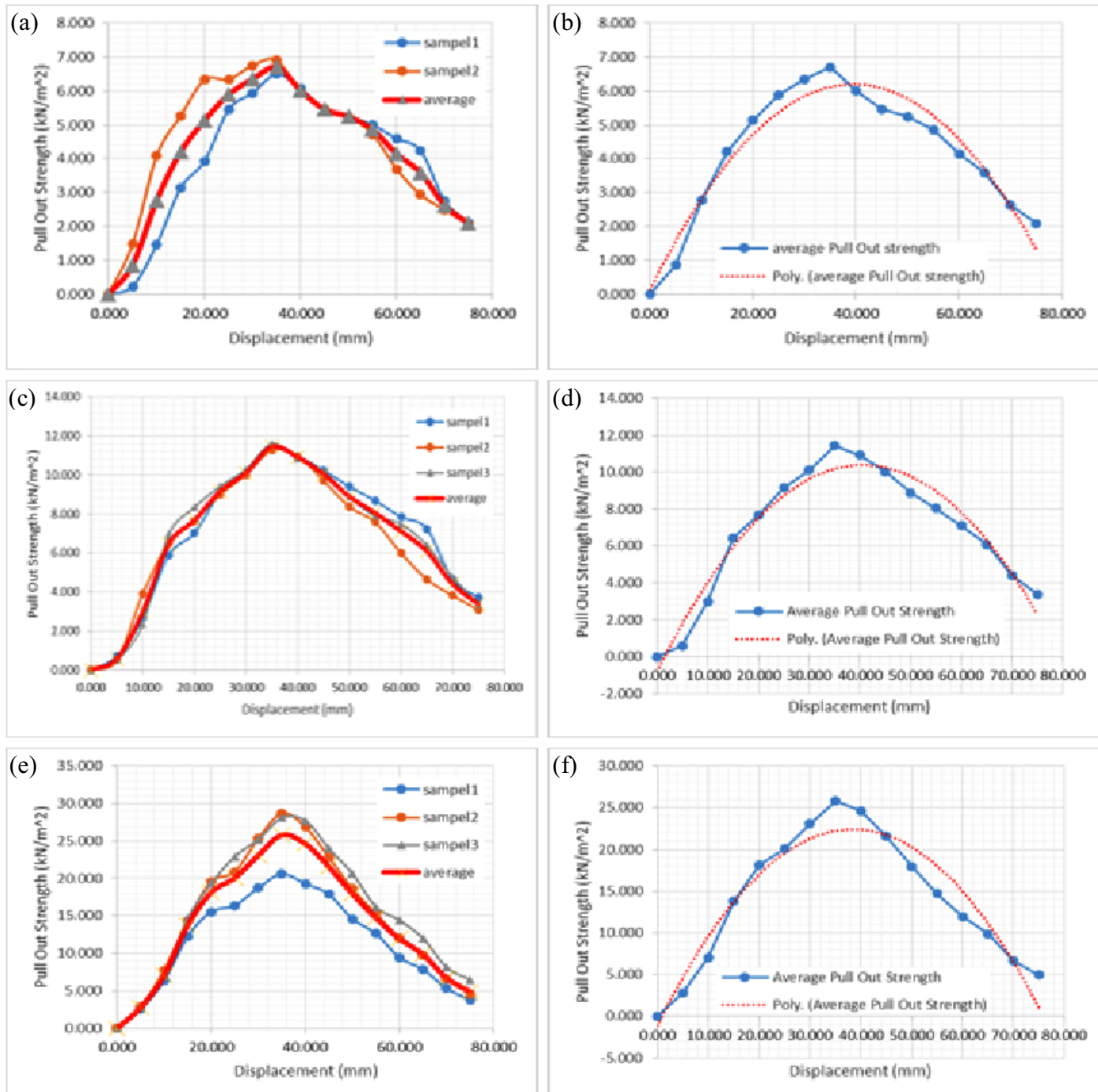

Figure 6. Maximum pull-out strength against vertical displacement curve for three replicates Pennisetum setaceum grass of planting period; (a) $1^{\text {st }}$ month, (c) $2^{\text {nd }}$ month and (e) $3^{\text {rd }}$ month.

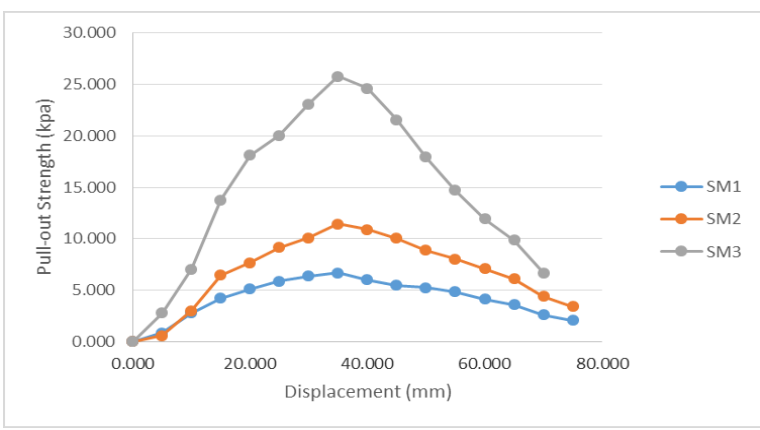

Figure 7. Average pull-out strength versus displacement for month 1, 2 and 3. 


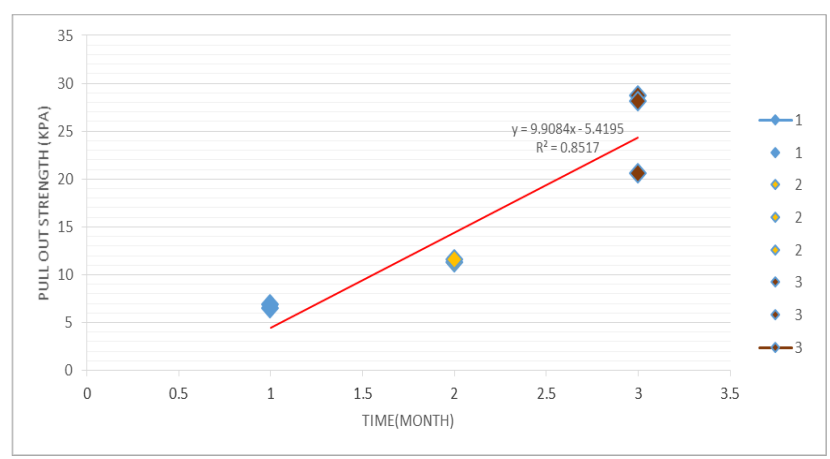

Figure 8. Max. pull-out strength versus growth period.
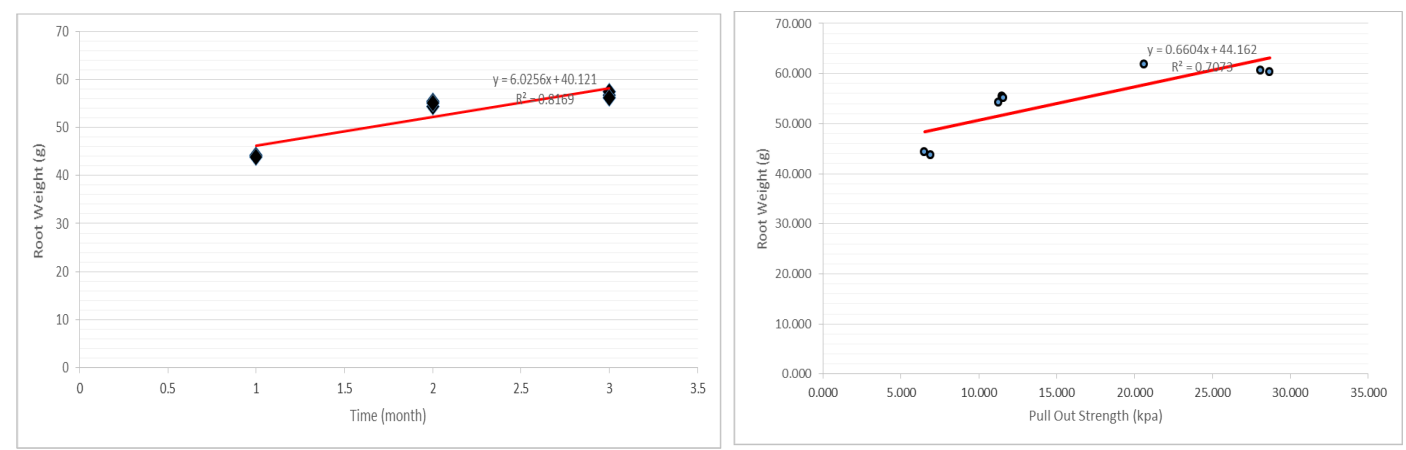

Figure 9. Root weight versus time. strength.

Figure 10. Root weight versus average pull-out
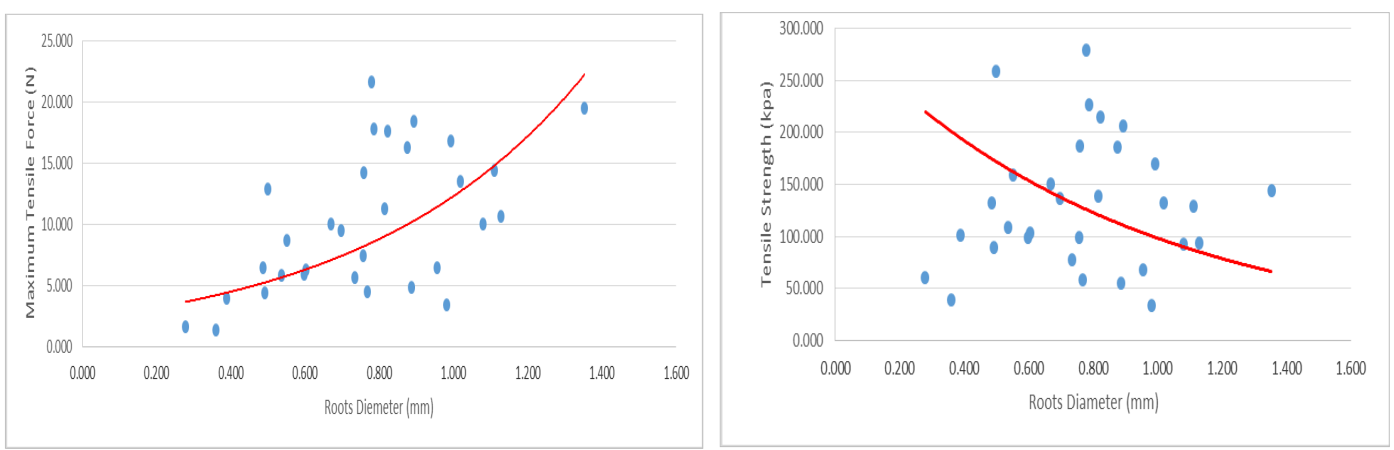

Figure 11. Max. tensile force versus root diameter.

Figure 12. Tensile stress versus root diameter.

\section{Conclusion}

It can be concluded that the pull-out strength of Pennisetum increasing over the time. This result was clearly demonstrated by series of pull-out tests that has been conducted in this study. Moreover, soil pull-out strength showed an increment due to plant growth in the root structure from time to time. It was due to the roots grip into the ground, giving some mechanical reinforcement effect resulted in strengthened of soil. As for the tensile strength, the value decreases with increasing root diameter. 


\section{Acknowledgement}

This research publication was funded by Multi-Disciplinary Research Grant (MDR), Vote no. U088. The researchers would like to thank staff on duty at Packaging Laboratory, Faculty of Mechanical and Manufacturing Engineering, Universiti Tun Hussein Onn Malaysia for the full permission given on the use of tensile strength equipment.

\section{References}

[1] M.S. Zainordin, N.A. Yusoff, T.N.T. Chik, M.A.H. Manap, Z. Sayuti, M.F. Abdullah, The study of soil-roots strength performance of soil slope by using guinea grass. J. Applied Mechanics and Materials, 802, 10-15, (2015).

[2] N.Osman, M.N. Abdullah, C.H. Abdullah, Pull-out and tensile strength properties of two selected tropical trees. Sains Malaysiana, 40(6), 577-585, (2011).

[3] M. Tosi, Root tensile strength relationships and their slope stability implications of three shrubs species in the Northern Apennines (Italy). Geomorphology, 87(4), 268-283, (2007).

[4] N. Pollen-Bankhead, A. Simon, and R.E. Thomas, The reinforcement of soil by roots: recent advances and directions for future research. Treatise on Geomorphology, 12(8), 107-124, (2013).

[5] S.B. Mickovski, L.P.H. van Beek, Root morphology and effects on soil reinforcement and slope stability of young vetiver (Vetiveria zizanioides) plants grown in semi-arid climate, Plant Soil, 324 (1-2), 43-56, (2009) 